\title{
Factores motivacionales intrínsecos en el uso de ePortfolios por el estudiantado en programas de Máster en Formación del Profesorado
}

\author{
Intrinsic Motivational Factors in the Use of ePortfolios by Students in Master's Degree \\ Programs in Education
}

\section{Fatores de motivação intrínsecos na utilização de ePortfolios por estudantes nos programas de Mestrado de Formação de Professores} \begin{abstract}
Universidad Complutense de Madrid mciesielkiewicz@villanueva.edu Universidad Autónoma de Madrid https://orcid.org/0000-0002-6724-4697 Universidad Complutense de Madrid https://orcid.org/0000-0002-9193-2916

Recibido • Received • Recebido: 26 / 07 / 2019

Corregido • Revised • Revisado: 27 / 01 / 2021

Aceptado • Accepted • Aprovado: 24 / 02 / 2021

Resumen: En esta investigación nos hemos propuesto analizar los factores intrínsecos que podrían motivar al estudiantado a crear y utilizar un ePortfolio. Las personas participantes de nuestro estudio son estudiantes que cursaron el Máster en Formación del Profesorado en tres universidades diferentes. Para los fines de este estudio se ha utilizado una encuesta cuantitativa online administrada a través de los formularios de Google. La motivación intrínseca se midió por medio de una evaluación basada en el Test de motivación intrínseca (IMI). Este instrumento midió las siguientes dimensiones: la competencia percibida al realizar el ePortfolio, el esfuerzo invertido al realizar esta tarea, el valor o utilidad que le han dado a su realización y la presión o tensión sentida por el estudiantado cuando hizo esta actividad. Nuestros datos indican que los factores intrínsecos que más motivan al alumnado son la apreciación del valor y utilidad del ePortfolio, seguidos de la percepción de la competencia adquirida.
\end{abstract}

Monika Ciesielkiewicz Madrid, España

David Méndez-Coca Madrid, España david.mendez@uam.es

Miriam Méndez-Coca Madrid, España mmendez@villanueva.edu

Palabras claves: E-portfolio; tecnología educativa; educación superior; percepción; motivación. 
http://doi.org/10.15359/ree.25-2.7

http://www.una.ac.cr/educare

educare@una.ac.cr

\begin{abstract}
In this paper, the authors intend to analyze the intrinsic factors that could motivate students to create and use an ePortfolio. The participants of the study were students of Master's Degree Programs in Secondary Education from three different universities in Spain. For this study, the authors used an online, quantitative survey administered through Google Forms. The intrinsic motivation was measured using a survey adapted from the Intrinsic Motivation Inventory (IMI). This instrument evaluated the following dimensions: the perceived competence while working with the ePortfolio, the effort invested in the task, the value or usefulness of this task, and the pressure or tension felt by the students while carrying out this activity. Our data show that the most important intrinsic factors motivating students are the appreciation of the value and usefulness of the ePortfolio, followed by the students' perception of the acquired competence.
\end{abstract}

Keywords: E-portfolio; educational technology; higher education; perception; motivation.

Resumo: Nesta pesquisa, propusemo-nos a analisar os fatores intrínsecos que poderiam motivar a estudantes a criar e usar um ePortfolio. Participaram do nosso estudo estudantes que fizeram o Mestrado em Formação de Professores em três Universidades diferentes. Para os fins deste estudo, foi utilizado um questionário quantitativo em linha, administrado através do Google Forms. A motivação intrínseca foi medida por meio de uma avaliação baseada no Teste de Motivação Intrínseca (IMI). Este instrumento mediu as seguintes dimensões: a competência percebida ao fazer o ePortfolio, o esforço investido na execução desta tarefa, o valor ou utilidade que deram para à sua realização e a pressão ou tensão sentida pelos estudantes quando completaram a atividade. Os nossos dados indicam que os fatores intrínsecos que os motivam mais são a valorização e a utilidade do ePortfolio, seguido da percepção das competências adquiridas.

Palavras-chave: E-portfólio; tecnologia educacional; educação superior; percepção; motivação.

\title{
Introducción
}

El ePortfolio o portafolio electrónico puede servir como una herramienta de aprendizaje y evaluación en el ámbito educativo, así como de presentación y reclutamiento a la hora de buscar trabajo. Incluye información sobre competencias, habilidades, formación formal e informal, así como experiencias personales y profesionales (Ciesielkiewicz, 2015).

Barberà Gregori y de Martín Rojo (2009) lo definen como "un instrumento metodológico y una metodología educativa en sí misma, lo que permite la evaluación del aprendizaje como proceso (evaluación formativa) y el producto final (evaluación sumativa) del aprendizaje del estudiante" (p. 20).

Numerosas habilidades y competencias se pueden desarrollar mediante el uso de portafolios electrónicos, por ejemplo, habilidades de aprendizaje activo, establecimiento de objetivos tanto para la educación como para la carrera profesional, aprendizaje independiente, aprendizaje colaborativo, competencias curriculares transversales, habilidades de comunicación interpersonal, habilidades de autoevaluación, habilidades de autovaloración, habilidades de 
http://doi.org/10.15359/ree.25-2.7

autorregulación, habilidades informáticas, preparación para el trabajo, aprendizaje permanente, autogestión, pensamiento de orden superior y autoconsciencia (Ciesielkiewicz et al., 2020; Kabilan y Khan, 2012; Zellers y Mudrey, 2007).

Según el informe de EDUCAUSE Center for Analysis and Research (Dahlstrom et al., 2013), más de un $50 \%$ de las instituciones de educación superior en EEUU han implantado algún tipo de ePortfolio. Además, en 2016 Association of American Colleges and Universities la incluyó entre las 11 prácticas docente más eficientes (High Impact Educational Practices) y de mayor impacto en la educación superior (Eynon y Gambino, 2017).

Asimismo, el gobierno australiano lo ha implementado en las universidades públicas y lo ha adaptado a los estándares de educación superior desarrollados por el Ministerio de Educación de Australia (Hallam y Creagh, 2010). Los materiales y documentos desarrollados en ese proyecto son de acceso abierto, y sirven de apoyo y ayuda para facilitar la implementación del ePortfolio en otros países.

Desgraciadamente, esta herramienta tan útil e innovadora es muy poco conocida en España, a pesar de ser objeto de varios proyectos europeos (EUfolio, 2020), cuyo patrocinio corre a cargo del Consejo de Europa. Asimismo, el informe de European Commission Joint Research Institute for Prospective Technological Studies (Redecker, 2013) menciona el ePortfolio como una de las estrategias de educación continua del futuro.

En EEUU los proyectos de ePortfolio son iniciativas propias de cada universidad tanto a nivel institucional, de programas, proyectos o profesorado que quieran implementarlo. Por otro lado, la organización profesional Ilamada The Association for Authentic, Experiential and Evidence-Based Learning creada en EEUU promueve la investigación sobre este instrumento metodológico y, anualmente, organiza congresos sobre el tema. Asimismo, existe un proyecto nacional Connect to Learning. Catalyst for Learning: ePortfolio Resources and Research (Connect to Learnig, 2019) patrocinado por el Ministerio de Educación de EEUU en el que participaron 24 universidades americanas tales como Boston's Northeastern University, San Francisco State, IUPUI, Manhattanville College, CUNY, entre otras, cuyos objetivos son: 1) compartir las buenas prácticas que les hayan resultado eficaces, 2) impulsar la investigación sobre el ePortfolio en la enseñanza, aprendizaje y evaluación, 3) ofrecer apoyo a las universidades interesadas en usar esta herramienta como metodología y práctica docente.

Asimismo, es importante destacar que el ePortfolio constituye un soporte de información digital, lo que supone una novedad respecto al curriculum vitae tradicional en papel.

Según el informe (Hart Research Associates, 2013), titulado It takes more than a major: Employer Priorities for College Learning and Student Success desarrollado por la Association of American Universities and Colleges, un $84 \%$ de los 314 entes empleadores consideraron el ePortfolio como una herramienta muy útil a la hora de seleccionar candidatos o candidatas para un 
http://doi.org/10.15359/ree.25-2.7

http://www.una.ac.cr/educare

educare@una.ac.cr

puesto de trabajo. Los resultados de una investigación llevada a cabo con 52 personas directoras de recursos humanos y 35 de colegios en el contexto español (Ciesielkiewicz, 2015, 2019), también lo corroboran, ya que el $80 \%$ de quienes participaron lo consideran una herramienta útil para obtener información más amplia y detallada sobre quienes postulan su candidatura para un puesto de trabajo.

Con la transformación digital que muchas empresas están llevando a cabo, será imprescindible que la comunicación entre postulantes a una oferta de empleo y los entes empleadores se realice a través de un canal tecnológico. El portafolio electrónico responde perfectamente a esta necesidad y facilitará, por una parte, el acceso de los candidatos y candidatas al mundo laboral y, por otra, la gestión de la información a las compañías.

El objetivo de esta investigación es estudiar los factores de la motivación intrínseca del estudiantado en el uso del ePortfolio. El equipo de autoría se propone analizar el valor, utilidad, e importancia del ePortfolio y el esfuerzo, la competencia, la presión o tensión sentida por el estudiantado al realizarlo, a través de una encuesta adaptada a los objetivos de la investigación. Este estudio puede contribuir a la comprensión de cómo estudiantes de posgrado perciben sus portafolios electrónicos y cómo el profesorado puede motivarles para promover su aprendizaje y crecimiento profesional a través de este medio.

\section{La investigación sobre la percepción del estudiantado acerca del ePortfolio}

El estudiantado reconoce el beneficio del ePortfolio como herramienta de aprendizaje según las últimas investigaciones realizadas por Klampfer y Köhler (2015) y Welsh (2012). Por su parte, Bolliger y Shepherd (2010) demostraron que el $85 \%$ de quienes participaron en su estudio estuvieron de acuerdo en que el uso de este aumentaba su interés por aprender. Igualmente, la investigación realizada por Wakimoto y Lewis (2014) concluyó que el 90\% de las personas participantes estaban de acuerdo en que el ePortfolio era beneficioso, aunque el $45 \%$ pensaba que la actividad, en sí misma, era confusa. Klampfer y Köhler (2015) analizaron los factores que afectan la motivación y la actitud hacia los ePortfolios y concluyeron que la utilidad percibida y la relevancia son los dos factores cognitivos y contextuales que más influyen en los niveles de motivación. Mobarhan et al. (2015) analizaron un factor adicional y comprobaron que la autonomía incrementa la motivación del estudiantado para dedicar su esfuerzo en desarrollar el portafolio electrónico. El estudiantado afirma que sus ganas por aprender aumentan debido al uso de esta herramienta (Klampfer y Köhler, 2015). Por otro lado, aquel estudiantado que manifiesta un bajo deseo por aprender ofrece una evaluación negativa de esta tarea. Asimismo, el nivel de calidad en la ejecución del ePortfolio viene determinado por el tipo y el nivel de motivación del estudiantado. Cheng y Chau (2013) demostraron que el estudiantado que presenta niveles altos de motivación, tanto intrínseca como extrínseca, realizan con mayor calidad las actividades de ePortfolio que aquel estudiantado que presenta un único tipo de motivación.

4 
Este instrumento metodológico puede beneficiar al estudiantado tanto durante su carrera académica como profesional; sin embargo, como advierten Eynon y Gambino (2017), su efectividad disminuye o incluso es nula cuando no se implementa correctamente. La creación de un ePortfolio no es un proceso simple y requiere preparación y formación dirigida tanto al profesorado como al estudiantado (Cheng y Chau, 2013). Harring y Luo (2016) describieron que cuandoel ePortfolio seimplementó por primera vezen su universidad, el estudiantado no percibió sus beneficios, sobre todo, no entendía la utilidad e impacto en su formación universitaria. Sin embargo, cuando los equipos investigadores examinaron el diseño del programa, identificaron los fallos y aplicaron los cambios y sugerencias, la percepción del estudiantado cambió. Gracias a las modificaciones, el estudiantado reconoció tanto la relevancia de esta tecnología en cuanto herramienta de aprendizaje como su potencial más allá de la formación universitaria.

El nivel de la competencia tecnológica del alumnado es también un factor importante que influye en su motivación. La adopción de nueva tecnología requiere de la motivación intrínseca del alumnado (Winne y Hadwin, 2012) y, a su vez, la competencia es un requisito previo para la motivación (Deci y Ryan, 2008). De manera más específica, Mobarhan et al. (2015) observaron que cuanto mayor es la competencia digital del estudiantado mejor es su percepción de esta herramienta. Igualmente, destacaron que contar con una buena infraestructura de TI y el sistema utilizado para el ePortfolio afecta positivamente la motivación del público usuario. ZainalAbidin et al. (2011) destacaron tres elementos determinantes en la evaluación positiva de esta tarea: que sea fácil de entender, que sea fácil de usar y que la infraestructura de TI funcione correctamente. Sin embargo, cabe señalar que Klampfer y Köhler (2015) no encontraron una correlación significativa entre la competencia digital o el interés para el uso de la tecnología con la motivación para usar esta herramienta. Además, Birks et al. (2016) realizaron un estudio en el que permitieron al alumnado escribir comentarios libres sobre los problemas que iban encontrando. Los problemas que encontraron con la plataforma tecnológica influyeron negativamente en su percepción del ePortfolio.

Diversos estudios destacan lo determinante que es la ayuda que otras personas prestan al estudiantado para que su percepción del ePortfolio mejore. Proveer de apoyo durante el proceso de creación del este se manifiesta como clave para el éxito de su implementación. En la investigación realizada por Wakimoto y Lewis (2014), el grupo de participantes puso mucho énfasis en la importancia del apoyo que recibió del personal de TI y de sus compañeros y compañeras. El noventa por ciento de este grupo de participantes evaluó positivamente el ePortfolio y destacó sus beneficios. Klamper y Köhler (2015) lo corroboran, ya que encontraron una correlación muy alta entre el apoyo recibido y la motivación para usar esta herramienta. En esta línea, la ayuda de sus pares y docentes fue también importante para una mayor efectividad de la autorregulación del aprendizaje (Welsh, 2012).

Por otro lado, existen datos contradictorios en cuanto a las percepciones del estudiantado acerca de la utilidad del ePortfolio como herramienta de búsqueda de trabajo y desarrollo 
http://doi.org/10.15359/ree.25-2.7

http://www.una.ac.cr/educare

educare@una.ac.cr

profesional. Wakimoto y Lewis (2014) realizaron una investigación con estudiantes de posgrado en psicología y programas de asesoramiento quienes tuvieron que crear portafolios electrónicos de prácticas profesionales. El objetivo de su ePortfolio era documentar las competencias profesionales para obtener la certificación y acreditación profesional en su campo. Dado que este era el propósito principal de la actividad, no es sorprendente que la mayoría de las personas participantes considerara que la herramienta era útil para la búsqueda de trabajo y el desarrollo profesional. De hecho, el 86\% de las personas participantes expresaron su intención de compartir el ePortfolio con sus futuros entes empleadores. Sin embargo, en un estudio similar (Birks et al., 2016) llevado a cabo con alumnado de posgrado y doctorado en enfermería, solo el 36\% admitió que les sería útil para buscar empleo y solo el $30 \%$ afirmó que seguirían utilizando este instrumento metodológico después de terminar sus estudios. Sin embargo, hay que mencionar que dicho alumnado denunció numerosos problemas con la plataforma tecnológica, lo que pudo influir en su percepción negativa de este como herramienta útil para su futuro profesional.

\section{Metodología}

Con el fin de investigar qué factores intrínsecos podrían motivar al estudiantado a crear y utilizar con éxito un ePortfolio, se utilizó un enfoque pragmático y cuantitativo. La motivación intrínseca se midió con una prueba basada en el Test de motivación intrínseca (IMI), que incluyó 22 ítems en forma de afirmaciones que el alumnado valoró según el modelo de escala Likert, de uno a siete. El Test de motivación intrínseca (IMI) se ha utilizado con éxito en varios estudios que miden la autorregulación y la motivación intrínseca (Deci y Ryan, 2008), entre ellos su validez fue confirmada por McAuley et al. (1989). Además, es un test ampliamente utilizado en estudios de motivación para medir el efecto de herramientas tecnológicas (Mendez y Sota, 2017; Mendez et al., 2018). Para los fines de este estudio, se utilizó una encuesta administrada a través de los formularios de Google.

La investigación es cuantitativa. La muestra fueron estudiantes del Máster de formación del profesorado de secundaria, bachillerato y formación profesional, en total 74 estudiantes que estaban cursando en ese momento en tres universidades a las que se tuvo acceso: 12 en la Universidad 1, 32; en la Universidad 2, y 30 en la Universidad 3. La investigación se llevó a cabo durante un semestre entre octubre de 2016 y febrero de 2017.

\section{Instrumento}

El Test de motivación intrínseca (IMI) que se pasó al estudiantado tenía 22 preguntas con escala Likert. La respuesta que debía escoger el alumnado era de 1 a 7 ante cada ítem. Esta es la escala completa: 1 - Muy en desacuerdo; 2 - En desacuerdo; 3 - De algún modo en desacuerdo; 4 - Ni de acuerdo ni de acuerdo; 5 - De algún modo de acuerdo; 6 - De acuerdo; 7 - Muy de acuerdo 
http://doi.org/10.15359/ree.25-2.7

Para adaptar el IMI a la presente investigación, se eligieron cuatro de las seis subescalas originales incluidas en el instrumento, a saber: la competencia percibida al realizar el ePortfolio, el esfuerzo realizado al realizar esta tarea, el valor o utilidad que le han dado a la realización del portafolio y la presión o tensión sentida por el estudiantado cuando lo hizo.

En todo el análisis la puntuación a partir de la cual se considerará positivo el resultado será 4. Por encima de 4 será positivo y por debajo será negativo para las afirmaciones en sentido positivo, en consecuencia, para las afirmaciones en sentido negativo será al revés, por debajo de 4 será una puntuación positiva y por encima será negativa.

\section{Resultados}

Las respuestas están organizadas en diferentes tablas según las dimensiones que mide el test utilizado. La Tabla 1 recoge los resultados ante los ítems relacionados con la competencia percibida por el estudiantado ante el ePortfolio realizado.

Tabla 1: Media de las respuestas del alumnado a las preguntas sobre la competencia percibida acerca del ePortfolio realizado

\begin{tabular}{|c|c|c|c|c|}
\hline Ítem & Universidad 1 & Universidad 2 & Universidad 3 & Media \\
\hline $\begin{array}{l}\text { Creo que he sido bastante bueno en las tareas } \\
\text { realizadas para el ePortfolio }\end{array}$ & $6,22 \pm 0,66$ & $4,72 \pm 1,19$ & $4,88 \pm 1,59$ & $4,97 \pm 1,37$ \\
\hline $\begin{array}{l}\text { Creo que he hecho las actividades del ePortfolio } \\
\text { bastante bien en comparación con otros/as estudiantes }\end{array}$ & $5,33 \pm 1,22$ & $4,08 \pm 1,02$ & $4,24 \pm 1,66$ & $4,30 \pm 1,35$ \\
\hline $\begin{array}{l}\text { Me sentí bastante competente después de haber } \\
\text { hecho las actividades de ePortfolio }\end{array}$ & $6,11 \pm 1,05$ & $4,17 \pm 1,25$ & $4,84 \pm 1,55$ & $4,65 \pm 1,47$ \\
\hline $\begin{array}{l}\text { Estoy satisfecho/a con mi desempeño con los } \\
\text { trabajos realizados para el ePortfolio }\end{array}$ & $6,44 \pm 0,73$ & $4,75 \pm 1,16$ & $4,84 \pm 1,62$ & $5,00 \pm 1,40$ \\
\hline He estado bastante hábil realizando las tareas & $6,22 \pm 1,09$ & $4,61 \pm 1,38$ & $4,92 \pm 1,47$ & $4,93 \pm 1,46$ \\
\hline $\begin{array}{l}\text { El ePortfolio fue una tarea que era incapaz de realizar } \\
\text { muy bien }\end{array}$ & $2,00 \pm 1,94$ & $2,83 \pm 1,65$ & $2,52 \pm 1,75$ & $2,61 \pm 1,72$ \\
\hline
\end{tabular}

Nota: Elaboración propia. Puntuación positiva a partir de 4 para las afirmaciones con sentido positivo y menor de 4 para las afirmaciones con sentido negativo. 
http://doi.org/10.15359/ree.25-2.7

http://www.una.ac.cr/educare

educare@una.ac.cr

Los resultados en general muestran que el estudiantado percibe su competencia al realizar el ePortfolio positivamente, todas las afirmaciones en sentido positivo están por encima de 4 y en la afirmación con sentido negativo está por debajo. La muestra 1 percibe muy positivamente su capacidad, mientras la muestra 2 la percibe de forma sensiblemente positiva. Además, las respuestas tienen una desviación típica baja, lo que asegura que muy poco estudiantado considera su competencia de forma negativa a la hora de enfrentarse con las tareas.

Los datos recogidos en la Tabla 2 son los de la segunda dimensión del test, cómo el alumnado considera que se ha esforzado durante la tarea realizada.

Tabla 2: Media de las respuestas del alumnado a las preguntas sobre el esfuerzo al realizar el ePortfolio

\begin{tabular}{|c|c|c|c|c|}
\hline Ítem & Universidad 1 & Universidad 2 & Universidad 3 & Media \\
\hline $\begin{array}{l}\text { He puesto mucho empeño en las tareas del } \\
\text { ePortfolio }\end{array}$ & $5,55 \pm 2,13$ & $4,50 \pm 1,54$ & $3,96 \pm 1,51$ & $4,44 \underline{1} 1,66$ \\
\hline $\begin{array}{l}\text { No me he esforzado mucho en hacer las } \\
\text { actividades de } \\
\text { ePortfolio bien }\end{array}$ & $1,66 \pm 1,32$ & $3,00 \pm 1,84$ & $3,52 \pm 1,73$ & $3,01 \pm 1,81$ \\
\hline $\begin{array}{l}\text { Me he esforzado mucho en realizar las } \\
\text { actividades de ePortfolio }\end{array}$ & $5,55 \pm 1,81$ & $4,50 \pm 1,82$ & $4,40 \pm 1,68$ & $4,60 \pm 1,64$ \\
\hline $\begin{array}{l}\text { Ha sido importante para mí hacer bien las } \\
\text { tareas de ePortfolio }\end{array}$ & $5,89 \pm 1,36$ & $4,03 \pm 1,71$ & $4,84 \pm 1,95$ & $4,56 \pm 1,85$ \\
\hline $\begin{array}{l}\text { He invertido mucha energía en la realización de } \\
\text { las actividades de ePortfolio }\end{array}$ & $5,00 \pm 1,66$ & $3,94 \pm 1,31$ & $3,76 \pm 1,45$ & $4,01 \pm 1,44$ \\
\hline
\end{tabular}

Nota: Elaboración propia. Puntuación positiva a partir de 4 para las afirmaciones con sentido positivo y menor de 4 para las afirmaciones con sentido negativo.

En general, los resultados muestran que se han esforzado al realizar el ePortfolio y han considerado una esta tarea como importante: en la mayoría de los casos la puntuación está por encima de 4, en la afirmación con sentido negativo está por debajo. No obstante, no han invertido mucha energía en esta tarea. La muestra 1 vuelve a tener los resultados más altos y las otras muestras han tenido resultados muy similares.

Los resultados de la dimensión acerca del valor de las tareas que realizan vienen recogidos en la Tabla 3. 
http://doi.org/10.15359/ree.25-2.7

http://www.una.ac.cr/educare educare@una.ac.cr

Tabla 3: Media de las respuestas del alumnado a las preguntas sobre la utilidad/valor acerca del ePortfolio realizado

\begin{tabular}{|c|c|c|c|c|}
\hline Ítem & Universidad 1 & Universidad 2 & Universidad 3 & Media \\
\hline Creo que aprender el ePortfolio es útil & $6,44 \pm 1,01$ & $4,69 \pm 1,91$ & $5,36 \pm 1,98$ & $5,16 \pm 1,91$ \\
\hline $\begin{array}{l}\text { Creo que el ePortfolio es una herramienta importante } \\
\text { porque puede ayudarme tanto como herramienta de } \\
\text { evaluación y enseñanza como en mi búsqueda de empleo }\end{array}$ & $6,55 \pm 1,01$ & $4,83 \pm 1,76$ & $5,36 \pm 2,02$ & $5,24 \pm 1,85$ \\
\hline $\begin{array}{l}\text { Estaría dispuesto a crear y usar ePortfolios nuevos si } \\
\text { me resulta útil en el futuro }\end{array}$ & $6,66 \pm 0,70$ & $5,47 \pm 1,18$ & $5,56 \pm 2,00$ & $5,66 \pm 1,52$ \\
\hline $\begin{array}{l}\text { Creo que realizar esta tarea me podría ayudar en mi } \\
\text { carrera profesional y a desempeñar mejor mi trabajo }\end{array}$ & $6,66 \pm 0,70$ & $4,58 \pm 1,73$ & $5,16 \pm 1,97$ & $5,06 \pm 1,84$ \\
\hline $\begin{array}{l}\text { Considero que realizar esta actividad puede ser de } \\
\text { provecho para mí }\end{array}$ & $6,33 \pm 0,87$ & $4,67 \pm 1,80$ & $5,36 \pm 1,98$ & $5,13 \pm 1,85$ \\
\hline Creo que es el ePortfolio es una actividad importante & $6,33 \pm 0,86$ & $4,11 \pm 1,75$ & $5,04 \pm 2,01$ & $4,73 \pm 1,90$ \\
\hline
\end{tabular}

Nota: Elaboración propia. Puntuación positiva a partir de 4 para las afirmaciones con sentido positivo y menor de 4 para las afirmaciones con sentido negativo.

El valor o la utilidad que le han dado a esta tarea ha sido significativamente positivo, es la dimensión que tiene un resultado más alto. En la muestra 1 existe solo un estudiante que ha contestado por debajo de 6 a las cuestiones sobre esta dimensión. También la muestra 3 tiene unos resultados por encima de 5 en todas las preguntas. Solo la muestra 2 es la que logra unos resultados un poco más bajos, pero por encima de 4 en todos los ítems.

A continuación, en la Tabla 4 referimos los resultados sobre la presión o tensión sentida por el estudiantado al realizar la tarea.

Tabla 4: Media de las respuestas del alumnado a las preguntas sobre la presión/tensión sentida al realizar el ePortfolio

\begin{tabular}{|c|c|c|c|c|}
\hline Ítem & Universidad 1 & Universidad 2 & Universidad 3 & Media \\
\hline $\begin{array}{l}\text { No me he sentido nada nervioso/a cuando estaba } \\
\text { realizando las actividades del ePortfolio }\end{array}$ & $5,88 \pm 1,90$ & $5,33 \pm 1,57$ & $5,16 \pm 1,93$ & $5,34 \pm 1,73$ \\
\hline $\begin{array}{l}\text { Me he sentido muy tenso/a cuando estaba realizando } \\
\text { las actividades del ePortfolio }\end{array}$ & $1,77 \pm 1,99$ & $2,25 \pm 1,30$ & $2,00 \pm 1,29$ & $2,10 \pm 1,38$ \\
\hline $\begin{array}{l}\text { Estaba relajado/a cuando estaba realizando las } \\
\text { actividades del ePortfolio }\end{array}$ & $6,55 \pm 0,53$ & $5,05 \pm 1,62$ & $5,32 \pm 2,03$ & $5,34 \pm 1,74$ \\
\hline $\begin{array}{l}\text { Estaba ansioso/a cuando estaba realizando las } \\
\text { actividades del ePortfolio }\end{array}$ & 1,44 & $2,47 \pm 1,34$ & $2,24 \pm 1,61$ & $2,26 \pm 1,43$ \\
\hline $\begin{array}{l}\text { Me sentía presionado/a cuando estaba realizando las } \\
\text { actividades del ePortfolio }\end{array}$ & $1,33 \pm 0,50$ & $2,81 \pm 1,69$ & $2,36 \pm 1,60$ & $2,46 \pm 1,61$ \\
\hline
\end{tabular}

Nota: Elaboración propia. Puntuación positiva a partir de 4 para las afirmaciones con sentido positivo y menor de 4 para las afirmaciones con sentido negativo. 
http://doi.org/10.15359/ree.25-2.7

http://www.una.ac.cr/educare

educare@una.ac.cr

Significativamente el estudiantado ha contestado a las preguntas sobre esta dimensión que no se ha sentido tenso ni presionado, más bien han realizado el ePortfolio de forma tranquila y relajada. Los resultados de las tres muestras indican claramente esto. En las preguntas que son al revés, para comparar con la escala de 1 a 7 se tendría que hacer la operación de ocho menos la puntuación reflejada en la tabla, eso arrojaría unos resultados en todas las preguntas para todas las muestras superiores a 5.

\section{Discusión}

El ePortfolio es considerado actualmente como una herramienta útil para el aprendizaje y evaluación (Basso-Aránguiz et al., 2018) por la mejora de habilidades de reflexión en el tiempo (Jenson, 2011) y de motivación para el aprendizaje (Mobarhan et al., 2015).

En el estudio presente se muestra que los mejores resultados se consiguieron en el valor y utilidad que le dan al portfolio electrónico (Tabla 3). Estos resultados corroboran los obtenidos en la investigación sobre el uso del ePortfolio recogida por Chen et al. (2012), con una muestra de 413 estudiantes de los cuales el $80 \%$ eran estudiantes de grado y el resto de máster, quienes tenían que valorar diferentes aspectos de la herramienta. Sin embargo, en este caso el estudiantado no hacía su ePortfolio sino que se les proporcionaba varios ejemplos de ePortfolios ya hechos, solo los utilizaban, el indicador que logró mejores resultados también fue la utilidad percibida de la herramienta. Los resultados logrados son coherentes con los de Mobarhan et al. (2015).

El nivel de comodidad y tranquilidad, a la hora de realizar las tareas con el porfolio electrónico, ha sido el segundo indicador de este estudio que consiguió una puntuación más alta (Tabla 4). Sin embargo, en la revisión de la bibliografía se encuentran algunas experiencias previas donde el alumnado experimenta un aumento de ansiedad al realizar una experiencia con ePortfolio (Erice y Ertas, 2011).

El ePortfolio es una herramienta que nos proporciona la tecnología. Si se compara con el uso de los recursos tradicionales en el aula como son el libro de texto y el cuaderno por parte de estudiantes de primaria y secundaria con un test de motivación muy similar basado en el IMI (Mendez et al., 2018), se puede observar que la motivación del alumnado de 11 años es superior en dos casos al estudiantado que empleó el ePortfolio; sin embargo, quienes usaron el portfolio muestran una mayor motivación que el estudiantado de 14 años en todas las facetas medidas.

Si ahora comparamos nuestros resultados con la motivación que genera el uso de las diversas aplicaciones que tiene la tableta para estudiar (Mendez et al., 2018) se puede observar que el alumnado más joven es el que está más motivado en todas las dimensiones, después los de secundaria, salvo en el valor y utilidad que le dan a la herramienta, por tanto, la utilidad que le está dando el futuro profesorado de secundaria al portfolio es muy alta. Hay que tener en cuenta que la relación entre la edad y la adopción de tecnología está influenciada por habilidades de aprendizaje, competencia tecnológica y niveles de ansiedad a la hora de utilizar estas herramientas (Czaja et al., 2006) y experiencias previas (Namlu, 2003). 


\section{Conclusiones}

El estudio presente desarrollado en tres universidades, para estudiar la motivación intrínseca del estudiantado en la utilización del portfolio electrónico a través de los factores que la causan, conforme a la teoría de la autodeterminación (Deci y Ryan, 2008) refleja una percepción positiva por parte del alumnado de su propia competencia para realizar tareas con el ePortfolio (Tabla 1) y de la utilidad del uso de esta herramienta en su trabajo como estudiante y en sus expectativas de uso en el futuro (Tabla 3 ). Además, los resultados muestran el interés del alumnado al realizar las tareas con esta herramienta (Tabla 2) y que no sintieron presión, ni ansiedad a la hora de incorporar e integrarla a sus tareas y experiencias (Tabla 4).

Aunque en todas las dimensiones de la motivación estudiadas se han obtenido buenos resultados, existen diferencias claras en las muestras y se aprecia unos mejores resultados en la dimensión de la valoración y utilidad del ePortfolio (Tabla 3) donde se obtiene una puntuación con una media de 5,17 sobre 7. Al compararlos con otros estudios, se puede observar que este dato es superior incluso a la motivación que tienen estudiantes de secundaria a la hora de trabajar con aplicaciones de la tableta.

Además, el análisis de los resultados muestra una percepción positiva de su propia competencia para realizar tareas con el ePortfolio (Tabla 1), con una media de 4,71 sobre 7. El alumnado no sintió presión ni ansiedad a la hora de incorporar e integrar este recurso (Tabla 4), valorando con 4,97 de media sobre 7, el grado de encontrarse cómodos y relajados en el uso de la herramienta. Los resultados más bajos se obtuvieron en el interés del alumnado para realizar las tareas con la herramienta (Tabla 2), donde se alcanza 4,32 de media sobre 7 que es un resultado también positivo.

En las prácticas de realización del ePortfolio se hace recomendable una cuidada selección del tipo de tecnología que se emplee, así como la atención a la ayuda que se presta durante la implementación de esta tarea para favorecer su realización y la motivación estudiantil hacia la tarea (Klampfer y Köhler, 2015; Mobarhan et al., 2015; Wakimoto y Lewis, 2014; Zainal-Abidinet al. 2011). La coordinación de estas prácticas en la universidad y la formación previa y continua sobre este instrumento metodológico favorecería diseños de estas actividades (Cheng y Chau, 2013; Harring y Luo, 2016).

Esta investigación aborda una cuestión crucial para los educadores y las educadoras que están implementando el ePortfolio, que ha demostrado ser una herramienta valiosa para la evaluación y el aprendizaje, así como para el desarrollo de la carrera. Entender cómo el estudiantado percibe sus portafolios puede servir al personal docente a promover la motivación estudiantil, así como su aprendizaje y crecimiento profesional a través de esta herramienta. Dicha investigación puede contribuir a la línea de investigación sobre este tema. Nuestros datos sugieren que el profesorado y las instituciones educativas que implementen el ePortfolio en sus asignaturas y programas deberían incluir estrategias que apoyen la motivación intrínseca, especialmente aquellas que manifiesten el valor y la utilidad de este instrumento. 
http://doi.org/10.15359/ree.25-2.7

http://www.una.ac.cr/educare

educare@una.ac.cr

\section{Declaración de Material complementario}

Este artículo tiene disponible, como material complementario:

-La versión preprint del artículo en https://doi.org/10.5281/zenodo.3765410

\section{Referencias}

Barberà Gregori, E. y de Martín Rojo, E. (2009). Portafolio electrónico: Aprender a evaluar el aprendizaje. Editorial OUC.

Basso-Aránguiz, M., Bravo-Molina, M., Castro-Riquelme, A. y Moraga-Contreras, C. (2018). Propuesta de modelo tecnológico para Flipped Classroom (T-FliC) en educación superior. Revista Electrónica Educare, 22(2), 1-17. https://doi.org/10.15359/ree.22-2.2

Birks, M., Hartin, P., Woods, C., Emmanuel, E., y Hitchins, M. (2016). Students' perceptions of the use of eportfolios in nursing and midwifery education. Nurse education in practice, 18, 46-51. https://doi.org/10.1016/j. $\underline{\text { nepr.2016.03.003 }}$

Bolliger, D. U. y Shepherd, C. E. (2010). Student perceptions of ePortfolio integration in online courses. Distance Education, 31(3), 295-314. https://doi.org/10.1080/01587919.2010.513955

Chen, M.-Y., Chang, F. M.-T., Chen, C.-C., Huang, M.-J., y Chen, J.-W. (2012). Why do individuals use e-Portfolios? Educational Technology \& Society, 15(4), 114-125. http://www.jstor.org/stable/jeductechsoci.15.4.114

Cheng, G. y Chau, J. (2013). Exploring the relationship between students' self-regulated learning ability and their ePortfolio achievement. The Internet and Higher Education, 17, 9-15. https://doi.org/10.1016/j. iheduc.2012.09.005

Ciesielkiewicz, M. (2015). El porfolio electrónico como recurso educativo y su impacto en la búsqueda de trabajo. Revista Tecnología, Ciencia y Educación, 2, 83-99. https://www.revistasocitec.org/index.php/TCE/article/ view/53

Ciesielkiewicz, M. (2019). Education for employability: The ePortfolio from school principals' perspective. On the Horizon, 27(1), 46-56. https://doi.org/10.1108/OTH-01-2019-0001

Ciesielkiewicz, M., Bonilla, C. F. y Santos Peñas, M. (2020). The acquisition of competences in transnational education through the ePortfolio. En Á. Herrero, C. Cambra, D. Urda, J. Sedano, H. Quintián y E. Corchado (Eds.), The 11th International Conference on EUropean Transnational Educational (ICEUTE 2020) (pp. 75-83). Springer. https:// doi.org/10.1007/978-3-030-57799-5

Connect to Learning. (2019). About C2L. http://c2l.mcnrc.org/about/

Czaja, S. J., Charness, N., Fisk, A. D., Hertzog, C., Nair, S. N., Rogers, W. A. y Sharit, J. (2006). Factors predicting the use of technology: findings from the Center for Research and Education on Aging and Technology Enhancement (create). Psychology and aging, 21(2), 333-352. https://doi.org/10.1037/0882-7974.21.2.333

Dahlstrom, E., Walker, J. D. y Dziuban, C. (2013). ECAR study of undergraduate students and information technology (Research Report). EDUCAUSE Center for Applied Research. 
http://doi.org/10.15359/ree.25-2.7

Deci, E. L. y Ryan, R. M. (2008). Self-determination theory: A macrotheory of human motivation, development and health. Canadian Psychology, 49(3), 182-185. https://doi.org/10.1037/a0012801

Erice, D. y Ertaş, A. (2011). The impact of e-portfolio on foreign language writing skills. Ankara Üniversitesi Eğitim Bilimleri Fakültesi Dergisi, 44(2), 73-94. https://doi.org/10.1501/Egifak 0000001225

EUfolio (2020). EUfolio Partner Organizations. http://eufolio.eu/partners/

Eynon, B., y Gambino, L. M. (2017). High-impact ePortfolio practice: A catalyst for student, faculty, and institutional learning. Stylus Publishing, LLC.

Hallam, G. y Creagh, T. (2010). ePortfolio use by university students in Australia: A review of the Australian ePortfolio Project. Higher Education Research \& Development, 29(2), 179-193. https://doi. org/10.1080/07294360903510582

Harring, K. y Luo, T. (2016). Eportfolios: Supporting Reflection and deep learning in high-impact practices. Peer Review: Association of American Colleges \& Universities, 18(3). https://www.aacu.org/peerreview/2016/ summer/Harring

Hart Research Associates (2013). It takes more than a major: Employer priorities for college learning and student success. Liberal Education, 99(2). https://www.aacu.org/sites/default/files/files/LEAP/2013 EmployerSurvey.pdf

Jenson, J. D. (2011). Promoting self-regulation and critical reflection Through writing students' use of electronic portfolio. International Journal of ePortfolio, 1(1), 49-60. http://theijep.com/pdf/IJEP19.pdf

Kabilan, M. K. y Khan, M. A. (2012). Assessing pre-service English language teachers' learning using e-portfolios: Benefits, challenges and competencies gained. Computers \& Education, 58(4), 1007-1020. https://doi. org/10.1016/j.compedu.2011.11.011

Klampfer, A. y Köhler, T. (2015). Learners' and teachers' motivation toward using e-portfolios. An empirical investigation. International Journal Continuing Engineering Education and Life-Long Learning, 25(2), 189-207. https://doi.org/10.1504/IJCEELL.2015.069867

McAuley, E., Duncan, T. y Tammen, V. V. (1989). Psychometric properties of the intrinsic motivation inventory in a competitive sport setting: A confirmatory factor analysis. Research quarterly for exercise and sport, 60(1), 4858. https://doi.org/10.1080/02701367.1989.10607413

Méndez, D. y Sota, J. (2017). La influencia del uso del tablet en la motivación en ciencias de los alumnos de primaria. Enseñanza de ciencias, extraordinario, 975-980. https://www.raco.cat/index.php/Ensenanza/issue/ view/25743

Mendez, D., Mendez, M. y Anguita, J. (2018). Motivation of 14-year-old students using tablets, compared to those using textbooks and workbooks. International Journal of Interactive Mobile Technologies (iJIM), 12(4), 86-96. https://doi.org/10.3991/ijim.v12i4.9203

Mobarhan, R., Rahman, A. A. y Majidi, M. (2015). Electronic portfolio motivational factors from students' perspective: A qualitative study. Knowledge Management \& E-Learning, 7(2), 265-279. https://doi.org/10.34105/j. kmel.2015.07.017

Namlu, A. G. (2003). The effect of learning strategy on computer anxiety. Computers in Human Behavior, 19(5), 565578. https://doi.org/10.1016/S0747-5632(03)00003-7 
http://doi.org/10.15359/ree.25-2.7

http://www.una.ac.cr/educare

educare@una.ac.cr

Redecker, C. (2013). The use of ICT for the assessment of key competences. Joint Research Centre of the European Commission Scientific and Policy Report. https://ec.europa.eu/jrc/en/publication/eur-scientific-and-technicalresearch-reports/use-ict-assessment-key-competences

Wakimoto, D. K. y Lewis, R. E. (2014). Graduate student perceptions of eportfolios: Uses for reflection, development, and assessment. The Internet and Higher Education, 21, 53-58. https://doi.org/10.1016/j.iheduc.2014.01.002

Welsh, M. (2012). Student perceptions of using the PebblePad e-portfolio system to support self-and peer-based formative assessment. Technology, Pedagogy and Education, 21(1), 57-83. https://doi.org/10.1080/147593 9X.2012.659884

Winne, P. H. y Hadwin, A. F. (2012). The weave of motivation and self-regulated learning. En D. H. Schunk y B. J. Zimmerman (Eds.), Motivation and self-regulated learning (pp. 309-326). Routledge.

Zainal-Abidin, W., Uisimbekova, A. y Alias, R. A. (2011, November). Post-implementation strategy for the adoption of e-portfolio among students in a Malaysian public university. En 2011 International Conference on Research and Innovation in Information Systems (ICRIIS), (pp. 1-5). IEEE. https://doi.org/10.1109/ICRIIS.2011.6125670

Zellers, M. y Mudrey, R. (2007). Electronic portfolios and metacognition: A phenomenological examination of the implementation of e-Portfolios from the Instructors' perspective. International Journal of Instructional Media, 34(4), 419- 430 